\title{
Object Image Linking of Earth Orbiting Objects in the Presence of Cosmics
}

\author{
Carolin Früh ${ }^{1, *}$, Thomas Schildknecht ${ }^{1}$ \\ Astronomical Institute, University of Bern, Sidlerstrasse 5, 3012 Bern, Switzerland \\ This is a preprint, final version of this article is available at Advances in \\ Space Research: http://dx.doi.org/10.1016/j.asr.2011.10.021
}

\begin{abstract}
In survey series of unknown Earth orbiting objects, no a priori orbital elements are available. In surveys of wide field telescopes possibly many nonresolved object images are present on the single frames of the series. Reliable methods have to be found to associate the object images stemming from the same object with each other, so-called linking. The presence of cosmic ray events, so-called Cosmics, complicates reliable linking of non-resolved images. The tracklets of object images allow to extract exact positions for a first orbit determination.
\end{abstract}

A two step method is used and tested on observation frames of space debris surveys of the ESA Space Debris Telescope, located on Tenerife, Spain: In a first step a cosmic filter is applied in the single observation frames. Four different filter approaches are compared and tested in performance. In a second step, the detected object images are linked on observation series based on the assumption of a linear accelerated movement of the objects over the frame during the series, which is updated with every object image, that could be successfully linked.

\footnotetext{
*Corresponding author

Email addresses: frueh@aiub.unibe.ch (Thomas Schildknecht), thomas.schildknecht@aiub.unibe.ch (Thomas Schildknecht)

${ }^{1}$ Astronomical Institute, University of Bern, Sidlerstrasse 5, 3012 Bern, Switzerland, phone: +41 764906535 , fax: +41316313869
} 
As results it has to be stated. Firstly, the automatic discrimination of cosmics and non-resolved object images on single frames remains difficult, especially for traces with a size of only a few pixel. Secondly, on observation series, the object image linking of GEO, GTO and MEO object images is reliable for a moderate amount of object images or cosmics on the frames if at least three object images are available per object and for four object images per object even in the presence of several hundred cosmics or images on the frames with a simple approach of an updated linear accelerated movement even over long observation series.

Keywords: image processing; space debris; optical observations

\section{Introduction}

The Astronomical Institute of the University of Bern (AIUB) performs surveys for unknown space debris object in geostationary (GEO), geostationarytransfer (GTO) orbits on a regular basis with the ESA Space Debris Telescope (ESASDT) located on Tenerife, Spain. The aim of those surveys is to detect faint uncatalogued space debris objects. In these surveys declination stripes are scanned in a so-called blind-tracking mode. In surveys of geostationary objects, the telescope is kept fixed in starring mode, for surveys of objects in geostationary-transfer orbits the apparent velocity of reference objects is tracked. A survey of the ESASDT consists of 15 to 30 frames, spaced by one minute. Also so-called targeted follow-up observations of known objects are performed to improve the initial orbit. Those consist of up to 11 frames, spaced by 30 seconds. In between exposures, the telescope is repositioned on the same star field. The ESASDT has a field of view of $0.7 \times 0.7$ degrees, resulting in two to three images per object in surveys and four to six images per object in follow-up observations. In both cases, the observations cover a time interval of two to three minutes.

Although a priori information of the target object is available in follow-up observations, the frames are processed with the same algorithms than the surveys, in order to also detect further objects visible on the frames and to link the object images of the target object in cases its orbital information is very poor.

Possibly many object images are displayed on the single frames of a survey observation series. In a first step traces of cosmic ray events are filtered 
on the single frames. In a second step, object images of the same object on the different frames of the series are linked together. The linked tracklets allow to extract the astrometric positions, which may lead to a first orbit determination in further processing steps.

\section{Cosmic Filter and Object Image Link Algorithms}

\subsection{Cosmic Filters}

If charged particles hit a charged coupled device (CCD) detector photoelectrons are released punctually ?. When the detector is read out, a cosmic ray impact leaves a trace similar to the one of photons. Not all charged particles hitting the detector are actually cosmic rays, but may also originate from weakly radioactive materials used in the construction of the CCD dewar, see e.g. ?. In the current work all charged particles resolving photo-electrons at the detector are subsumed under the term cosmics.

A standard procedure in dealing with cosmics in astronomic imaging is, to stack several images. The cosmics are filtered out because they are singular events irregularly spread over the CCD frame. This technique has the additional side effect that the signal to noise ratio of the observed object is increased ?. But stacking requires a precise alignment of the frames relative to the observed objects. For a successful stacking the images have to be aligned relative to the object's motion, an alignment with the stars would filter out the object images of fast moving objects. In surveys, when searching for new objects, the object's position on the frame and their exact motion are unknown. Therefore, possible ranges of object velocities and inclinations have to be assumed and many different stackings of the same frames have to be checked and the results cross-checked. Such an approach is suggested and implemented by T. Yanagisawa at the Mount Nyukasa observatory of the Japanese Aerospace Exploration Agency (JAXA) ?. This method is computationally intensive and time consuming and not (yet) feasible for real-time processing. Real-time processing is required, when tasking follow-up observations within the same night. Additionally, stacking poses limits on the object's orbits and its apparent movement at a very early processing step already. Therefore, in the ESASDT processing a filter approach is preferred on the single frames for cosmic rejection. A cosmic filter should be fast, and allow to distinguish cosmics from actual object images. A filter approach acting on single frames is only feasible if the pixel scale is small enough to 
allow for such a discrimination, i.e., when the object images and cosmics are displayed over a couple of pixels on the frame, to allow to determine distinguishing features.

Four different approaches have been used:

A so-called contrast filter has been developed, which filters cosmics via a threshold value for the ratio between intensity value of the brightest pixel $\mathrm{i}_{\text {peak }}$ and and the mean intensity of the four surrounding ones $i_{\text {sur }}$, calibrated with the background intensity $\rho$ :

$$
\text { threshold }=\frac{\mathrm{i}_{\text {peak }}-\text { noise } \cdot \sqrt{\sigma_{1}}-\rho}{0.5\left(\mathrm{i}_{\text {sur }}+\text { noise } \cdot \sqrt{\sigma_{2}}\right)-\rho}
$$

with

$$
\begin{gathered}
\sigma_{1}=\sigma_{\rho}^{2}+\text { gain } \cdot \max \left(\mathrm{i}_{\text {peak }}, \rho\right) \\
\sigma_{2}=\sigma_{\rho}^{2}+\operatorname{gain} \cdot \max \left(\mathrm{i}_{\text {sur }}, \rho\right)
\end{gathered}
$$

with $\sigma_{\rho}$ being the standard deviation of the improved background intensity, and gain being the value for the camera gain. The threshold value is determined empirically.

A so-called object image class filter is based on six threshold values. Those threshold values are determined empirically for five different object image classes. The threshold values are combined equally weighted. An object image class is defined as the number of pixels an object image candidate has on the frame:

$$
\begin{aligned}
\text { threshold }_{1} & =\mathrm{i}_{\text {peak }}-\rho & \text { threshold }_{2} & =\mathrm{i}_{\text {all }}-\rho \\
\text { threshold }_{3} & =\frac{\mathrm{i}_{\text {all }}}{\mathrm{i}_{\text {peak }}} & \text { threshold }_{4} & =\frac{\mathrm{FWHM}_{x}}{\mathrm{FWHM}_{y}} \\
\text { threshold }_{5} & =\frac{\mathrm{FWHM}_{x}}{n_{\text {pix }}} & \text { threshold }_{6} & =\frac{\mathrm{FWHM}_{y}}{n_{\text {pix }}}
\end{aligned}
$$

whereas, $i_{\text {peak }}$ is the intensity of the brightest pixel, $i_{\text {all }}$ the mean of the intensity of all pixel of the object image, $\rho$ the improved background intensity, $\mathrm{FWHM}_{x}$ and $\mathrm{FWHM}_{y}$ an approximation of the FWHM in x- and y-direction with respect to the pixel coordinates, and $n_{\text {pix }}$ the number of pixel belonging to the candidate image on the frame. The FWHM is approximated by counting the number of pixels in $\mathrm{x}$ - and $\mathrm{y}$-direction of the pixel coordinates, 
which are above the mean intensity of the whole object image in relation to all pixels of the object image. This approximative calculation of the FWHM is preferred over a Gaussian fit ?. The computational load of a Gaussian fit is higher, which affects real-time processing, but more importantly, a Gaussian fit presupposes an approximately Gaussian or Gaussian elongated brightness distribution of the object images on the frames. This assumption leads to wrong results for highly distorted images or for images partly below signal to noise level.

Two classical noise sensitive edge detection filters were used, Sobel and Prewitt filters. To ease the computational burden and since the candidate images on the frames are already detected, not the complete frame is filtered with the kernels but only the smallest pixel-box containing the object image, padded with a two pixel sized background border. The kernel for the Sobel filter are ?:

$$
\begin{aligned}
& \mathbf{G}_{\mathbf{x}}=\left(\begin{array}{ccc}
1 & 0 & -1 \\
2 & 0 & -2 \\
1 & 0 & -1
\end{array}\right) \quad \mathbf{G}_{\mathbf{y}}=\left(\begin{array}{ccc}
1 & 2 & 1 \\
0 & 0 & 0 \\
-1 & -2 & -1
\end{array}\right) \\
& \mathrm{G}=\sqrt{\mathrm{G}_{\mathrm{x}}+\mathrm{G}_{\mathbf{y}}}
\end{aligned}
$$

The kernel of the Prewitt filter are ?:

$$
\begin{aligned}
& \mathrm{G}_{\mathbf{x}}=\left(\begin{array}{ccc}
-1 & 0 & 1 \\
-1 & 0 & 1 \\
-1 & 0 & 1
\end{array}\right) \quad \mathrm{G}_{\mathbf{y}}=\left(\begin{array}{ccc}
-1 & -1 & -1 \\
0 & 0 & 0 \\
1 & 1 & 1
\end{array}\right) \\
& \mathrm{G}=\sqrt{\mathrm{G}_{\mathrm{x}}+\mathrm{G}_{\mathrm{y}}}
\end{aligned}
$$

The gradients in the $\mathrm{x}$ - and $\mathrm{y}$-direction are summed up.

All filters have been tuned conservatively using ten nights of the ESASDT observation campaigns between January and July 2006.

\subsection{Object Image Linking}

Even after filtering, possibly many different object image candidates (true object images of many different objects and left over cosmics) are displayed and it has to be decided, which object images in fact belong to the same object. This process is called linking of the object images and positions, respectively. The appearance of the object on the single frames can vary 


\section{$\cdot 1 \cdot+\cdot \cdot+$}

Figure 1: Five images spaced by 30 seconds of the same object observed with the ESASDT in January 2006.

heavily, as Fig.1 shows, in which the same object is displayed on five images of one series, spaced by 30 seconds only. Single object images can be missing although the object was in the field of view during the exposure, in the following called gap, because belong signal to noise level or in front of a star image. A correlation of the single unlinked object images with a catalogue, as done e.g. by ?, is not recommended: Firstly, the correlation itself can be much improved in taking apparent velocity information into account?, especially when dealing with optical observations. Secondly, no unknown objects cannot be determined from unlinked images only.

With the current observation strategy of the ESASDT, as explained in the introduction, two to three object images per object in GEO and GTO survey observations spaced by one minute, and four to six object images per object in follow-up observation series spaced by 30 seconds are available. In both cases the observations cover a time interval two to three minutes per object. A linear accelerated movement of the objects over the frames during the short observation series is assumed in J2000.0 space-fixed topocentric reference system. Two object image candidates on two subsequent frames are preliminarily linked and their apparent movement is calculated in standard coordinates in a tangent plane on the (topocentric) celestial sphere ?. This has the advantage to be independent of the specific observation scenario. This movement is used to define so-called allowed regions, in which the next object image is expected. An allowed region is a confidence region around the point of the expected next object image position in the tangent plane. The allowed region is defined by a deviation limit in the absolute value of the velocity and the direction of the velocity determined from the first two preliminarily linked object images to a possible third object image. If no object image is found in the allowed region, a gap can be tolerated. The allowed region is broadened up on the subsequent frame necessarily. If an object image is found in the allowed region, it is added to the preliminary linked tracklet and the apparent velocity is updated, see Fig.2. 


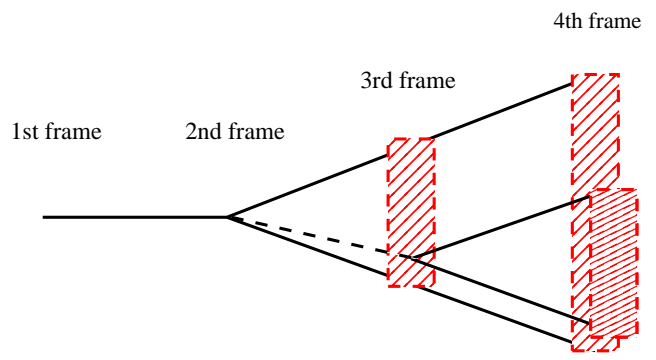

Figure 2: Linking object images on four frames. The boxes mark the allowed region.

In a next step, so-called cross linking conflicts are resolved: Cross-links are object images, which are linked in more than one tracklet. In a first step, all cross-links are allowed, and it is noted to which of possibly many different tracklets the single images belong. In a second step, the length of the tracklets, cross-linked by the same object image, are compared. The tracklet with the largest length, that is, the one containing of the most object images, keeps, the cross-linked image, all others are dissolved into single unlinked object images again. If two tracklets with the same length are cross-linked, the similarity of the cross-linked image with the preceding and following image within each tracklet is evaluated: The values of the main axis of inertial tensors of the preceding and following image are subtracted from the corresponding ones of the cross-linked image. The tracklet with the larger differences is dissolved. The procedure is iterated until no crosslinks occur any more. The remaining images are checked for new links of the newly dissolved images again. In a final step, links are made, in cases, if only two object images are available: They are linked if they fall within an apparent velocity and pseudo-inclination limit and if their inertial tensor fulfill a similarity criterion. The link via pseudo-inclination and apparent drift is necessary, since sometimes only two object images are available.

To determine the apparent drift $D$, and pseudo-inclination $I$ limit, the raw pixel coordinates are transformed into right ascension/declination $(\alpha / \delta)$ and azimuth/elevation $(a z / e l)$ using the pointing direction of the telescope:

$$
D=\frac{\sqrt{\left(a z_{2}-a z_{1}\right)^{2} \cos ^{2}\left(e l_{1}\right)+\left(e l_{2}-e l_{1}\right)^{2}}}{\left(t_{2}-t_{1}\right)}
$$




$$
I=\arctan \left(\frac{\delta_{2}-\delta_{1}}{\left(\alpha_{2}-\alpha_{1}\right) \cos \left(\delta_{1}\right)}\right)
$$

Currently a limit for the drift of 15 arcseconds per second and an inclination limit of 25 degrees is used. This allows to detect objects in a GEO region but puts significant limits on detectability of all other orbits. To link only two images of objects in other orbits, different drift and inclination intervals have to be chosen. The values of the main axis of the inertial tensor of the two images are allowed to deviate by less than 20 percent. The latter criterion is only applied if both images are brighter than 800 ADU, to avoid misinterpreting the inertial tensor of faint disintegrated traces close to the signal to noise level.

40 manually selected tracklets, which were linked only via the apparent drift and inclination limit and were manually corrected or were linked by hand, were evaluated in order to find empirical limit values for the deviations in absolute velocity and direction of velocity. The tracklets where picked randomly from the ESASDT campaigns of the first months of 2006. Only tracklets containing many object images were selected; the average was 4.5 images per tracklet. The absolute value of the apparent velocity calculated from the first two object images be $\left|v_{0}\right|$ and the absolute value of the apparent velocities calculated from the subsequent object image pair within the set be $\left|v_{i}\right|$, the difference in the direction of the velocity vector in the tangent plane be $\varangle v$; the expectation value and standard deviation of the velocity limit are determined as the following:

$$
\begin{gathered}
\left\langle\left|v_{i}\right| /\left|v_{0}\right|\right\rangle=0.185 \% \quad \sigma_{\left|v_{i}\right| /\left|v_{0}\right|}=0.156 \% \\
\langle\varangle v\rangle=0.001 \mathrm{rad} \hat{=} 0.063 \mathrm{deg} \\
\sigma_{\varangle v}=0.001 \mathrm{rad} \hat{=} 0.063 \mathrm{deg}
\end{gathered}
$$

\section{Performance with Real Observations}

The frames of the ESASDT are $2 \times 2$ binned in order to reduce the minimum signal-to-noise level for detectable objects. At the observation site of the ESASDT the seeing is normally larger than the pixel scale, therefore a binning is advantageous. The cosmic filters have been tested with ten survey observation series and three follow-up series of the night of August 25, 2006. The results are summarized in Table 1. In the appendix a few examples are 


\begin{tabular}{llllll} 
& Truth & Contrast & Obj. Class & Sobel & Prewitt \\
\hline object images & $\mathbf{9 6}$ & 84 & 85 & 84 & 79 \\
cosmics & $\mathbf{1 5 2 7}$ & 679 & 321 & 917 & 889 \\
obj. rate & $\mathbf{1 0 0 \%}$ & $87.5 \%$ & $88.5 \%$ & $87.5 \%$ & $82.2 \%$ \\
cosmic rate & $\mathbf{0 \%}$ & $55.5 \%$ & $79.0 \%$ & $36.4 \%$ & $41.8 \%$ \\
\hline
\end{tabular}

Table 1: Number of detected object images and cosmics on ten observation series of the ESASDT taken on August 25, 2006: Truth and filtered results.

shown of the detected object image candidates and the ones still present after filtering by the different algorithms.

The performance of rejecting the most cosmics is best for the contrast and object class filter. All filters misidentify true object images as cosmics, although they have been tuned as conservatively as possible. The Sobel filter is slightly superior to the Prewitt filter in the tested setup. But not all filters do misinterpret the same object images as cosmics, as a few examples listed in the appendix clearly show: The object class filter misinterprets the bright and large object images with more than 30 pixels (e.g. Fig. A.11), or object small object images with very few pixels, with a high peak intensity (e.g. Fig. A.8 and A.10). The latter cases could be as well cosmics as real object images, by eye inspection they were judged to be more likely object images. The contrast filter algorithm misinterprets mostly the object images with a small number of pixels, similar to the object class filter, as e.g. Fig. A.9 and A.10 show. The filter also misinterprets object images with medium pixel size, see, e.g., Fig. A.8. Here, the peak intensity of this object image is relatively high, but it clearly is an object image.

The performance of the linking algorithm is tested on two nights of the Tenerife campaign 2006 (January 26 and August 25) were used as an example. Table 2 shows the results.

The link of two object images is prone to mistakes. If more than two object images are available, the link is reliable. Only, two wrong sets have been linked with more than two object images. With those tracklets it was possible with both sets to determine a first orbit, but judging the single images 


\begin{tabular}{ll}
\hline sets & 781 \\
\hline correct sets & 158 \\
\hline correct sets $\geq 3 \mathrm{im}$ & 121 \\
\hline wrong sets $\geq 3 \mathrm{im}$ & 2 \\
\hline images per correct set & 4.19 \\
\hline manually corrected & 0 \\
\hline high inclination & 5 \\
\hline
\end{tabular}

Table 2: Tracklet linking of two nights of the Tenerife Campaign 2006 where analyzed with in total 117 observation series.

from eyesight, it was decided that they seem to show cosmics. No manual corrections needed to be applied to the sets itself, no images of different objects have been linked and no cosmics have been embedded in a set of more than two real object images. Restrictions on the orbit of link-able objects are weak enough to allow to link the images of objects, which are in high inclination orbits.

\section{Discussion of Methods}

\subsection{Limitations of Possibilities by the Cosmic Filter}

None of the cosmic filters is perfect when the aim is not to loose any object images. Filters which are based on many empirical parameters are highly dependent on the specific camera and have to be carefully re-tuned with every hardware change. In the step of linking object images it has to be assumed that a considerable amount of cosmics is still present.

\subsection{Random Links}

In the presence of left over cosmics after filtering and also possible many different object images displayed on the single frames, the probability of random (wrong) links with the current algorithm needs to be investigated. Observation series are assumed consisting of single frames containing a number of $q$ candidate object images. The probability of random links of tracklets consisting of $m$ candidates is investigated. The allowed number of gaps is assumed to be $n$. A square field of view is assumed. The probability for a random candidate linking, i.e., the probability that tracklets are detected, 


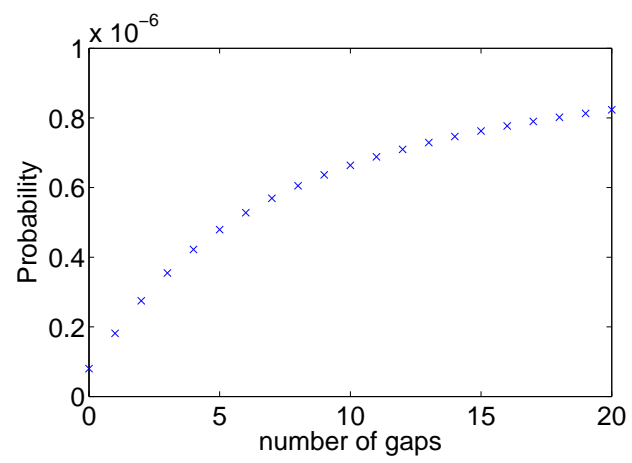

Figure 3: Probability of random links of tracklets of four candidate object images as a function of the number of allowed gaps within the set.

although no object is displayed on the frames, is estimated: For the estimation the candidates of a whole observation series are added up in a squared single frame, called summary frame in the following, with a given field of view (FOV). The density of candidates within this summary frame is calculated. The size of all allowed regions for a tracklet with a given number of images and gaps is determined under the premise that the whole tracklet (including gaps) can be displayed in the summary frame. This is in accordance with the ESASDT observation strategy. The probability to find a candidate in the allowed region with the given density of candidates is evaluated, which leads to the following expression:

$$
P \leq \sum_{i=0}^{n}\left(\frac{8}{(i+m-1)^{2}} \cdot\left(\left|v_{i}\right| /\left|v_{0}\right|\right)_{\text {limit }} \cdot \sin \left(\varangle v_{\text {limit }} / 2\right) \cdot q \cdot(i+1)\right)^{m-2}
$$

for $m \geq 3$.

The probability is slightly overestimated, especially in long observation series, since the density of candidates on the single observation frames is significantly lower than on the summary frame.

If 30 candidates are assumed on every frame, the probability of random links is $3.12 \cdot 10^{-3}$, if three gaps are allowed. If 300 candidates are assumed on every frame the probability is $2.52 \cdot 10^{-2}$, if two gaps are allowed. If only one gap is allowed the probability already decreased to $1.80 \cdot 10^{-3}$ even for 300 object images on each frame. If only one more object images is linked, so four in total, the probability of random links decreases by more than one order of magnitude. Figure 3 shows the probability for linking four candidates 


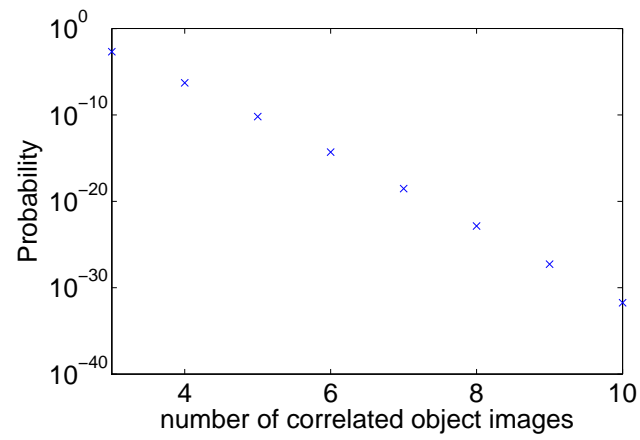

Figure 4: Probability of random linking as a function of the number of candidate object images in the tracklet (logarithmic scale).

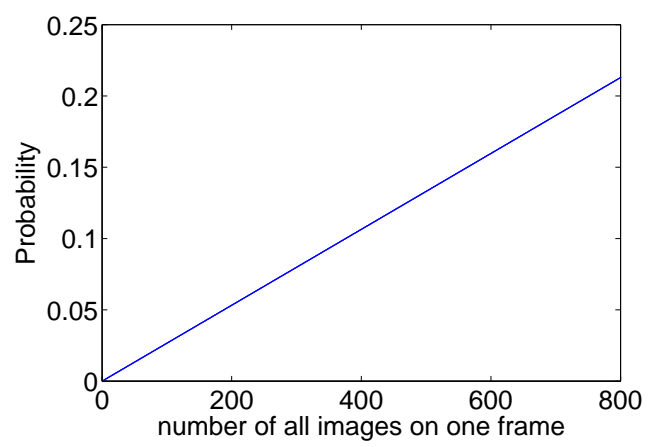

Figure 5: Probability of random links as a function of the number of candidate object images on one frame, (a) for linking of three candidates with a maximum number of two allowed gaps. 


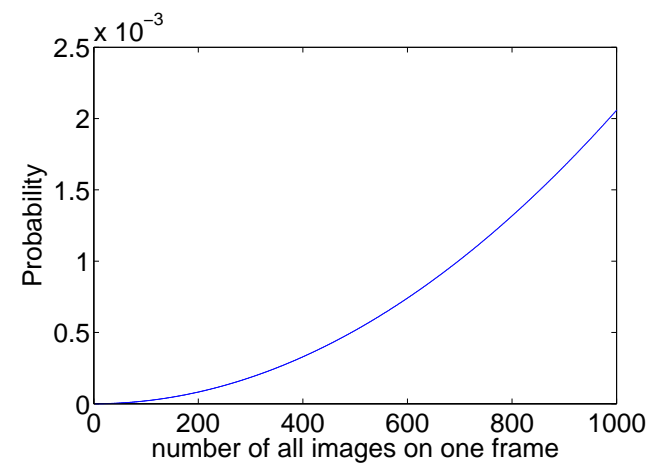

Figure 6: Probability of random links as a function of the number of candidate object images on one frame for linking of four candidates with a maximum number of six gaps.

into a tracklet as a function of the number of gaps allowed for 30 candidates per frame. The probability grows slowly with the number of allowed gaps. Figure 4 shows the probability of random links as a function of the number of candidates within the tracklet. The probability of random links rapidly decreases with every candidate added to the tracklet. Figure 5 and 6 shows the crucial dependence on the total number of candidates on each frame for tracklets with three or four candidates with a maximum of two and six allowed gaps, respectively. The probability for random links of candidates is generally higher for tracklets with only three candidates. The probability for random links grows linear for tracklets of the candidates and quadratically for tracklets with four candidates with the number of candidates images on the frames.

\subsection{Limits Imposed by Assumption of Steady Linear Apparent Motion}

The algorithm links object images based on the assumption of constant linear apparent motion of the objects over the frames during the observation series, but the velocity vector is updated with every successful link. Several orbits were analyzed to study the limitations imposed by this assumption, they are listed in Table 3. For all objects, geocentric ephemerides are determined over a time interval of 24 hours with a spacing of one minute. The ephemerides were transformed to the topocentric position of the ESASDT. It was assumed that all objects are visible during 24 hours, i.e., that the earth is transparent. This assumption has been made so the linking process is not 


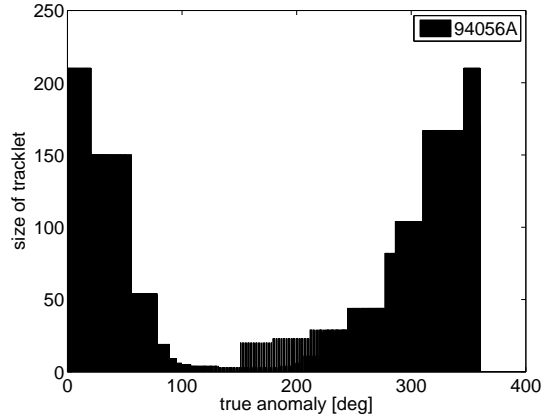

(a)

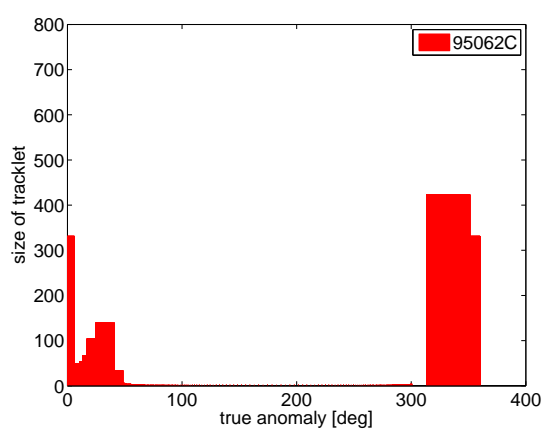

(c)

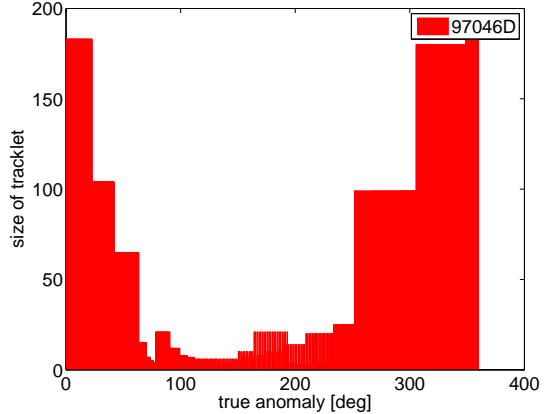

(b)

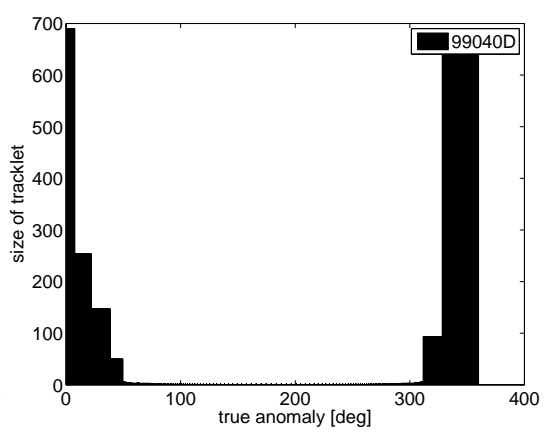

(d)

Figure 7: Length of the tracklets in minutes and amount of single images respectively, as a function of true anomaly of the object (a) 94056A, (b) 97046D, (c) 95062C and (d) 99040D . 


\begin{tabular}{llll} 
COSPAR & Incl. (deg) & Ecc. & Tracklets \\
\hline GEO & & & \\
\hline 05049B & 0.3184 & 0.0001 & 7 \\
90102A & 12.8753 & 0.0002 & 8 \\
91010F & 12.2318 & 0.0015 & 8 \\
\hline GTO & & & \\
\hline 94056A & 14.9243 & 0.5024 & 88 \\
97046D & 14.5544 & 0.4790 & 87 \\
\hline 95062C & 3.1615 & 0.8199 & 90 \\
99040D & 33.4316 & 0.8098 & 91 \\
\hline GPS & & & \\
\hline 84097A & 62.2247 & 0.0110 & 19 \\
85093A & 62.9986 & 0.0170 & 21 \\
\hline LEO & & & \\
\hline 58002B & 34.2430 & 0.1848 & 414 \\
\hline
\end{tabular}

Table 3: Test objects and Number of Tracklets within 24 hours.

limited by visibility constraints, but by the algorithm only. The topocentric ephemerides are transformed in so-called standard coordinates. A projection center has to be chosen for the transformation. In a real observation scenario this center is given by the pointing of the telescope. In the simulation, the topocentric viewing direction of the first ephemerides position is chosen. An unlimited field of view is assumed in the test setup: no new pointing is enforced by the object running out of the field of view. The theoretical telescope is assumed to be repositioned only, each time the algorithm is not able to connect the next object image to the current tracklet (because the linearity condition is violated, limit of the method is reached). After repositioning the object position is again in the center of the tangent plane. Only linking in forward direction is performed. If no third object image can be linked, the first two object images remain linked and are counted as one tracklet in the following. For the GEO objects not more eight tracklets have been formed over $24 \mathrm{~h}$ hours, for the objects in different inclination orbits. For the objects in high eccentricity orbits, 88 and 95 tracklets have been formed. But the length of the tracklets is highly dependent on the anomaly, as Fig.7 shows. For observations around the apogee the length of the track- 
lets is of the same order as for GEO objects. For the GTO objects with moderate eccentricities all tracklets with anomalies of over 300 and below 50 degrees are longer than 80 minutes. For the two objects in high eccentricity orbits tracklets longer than 150 minutes occur for anomalies larger than 320 and below 30 degrees. For the GPS satellites about 20 tracklets have been formed, due to the small eccentricities no anomaly dependency occurs. For the LEO satellite 414 tracklets have been formed. The algorithm would have to be adjusted to a curved movement to be suitable for the LEO regime.

\section{Conclusions}

Both algorithms are implemented and used in the routine processing of GEO and GTO surveys of the ESASDT, in order to detect new space debris objects. The following concluding remarks can be made:

It is possible to filter cosmics on the single observation frames. Classical edge detection filters appear to be inferior to empirical models in the detection of small pixel sized object images close to the signal to noise level, but deliver good results for bright large pixel object images. Empirical contrast filters deliver good results for faint object images.

Assuming a linear steady accelerated motion for the movement of the objects over the frames without further constraints allows to reliably link object images. As long as more than two object images are available even with the tolerance of missing object images, and in the presence of cosmics and several hundred of object image candidates, reliable linking with a false rate of below 0.5 percent is possible.

The linear accelerated motion, which is updated with every linked object image is a valuable approach in the link of images of GEO, and MEO objects. GTO object images are linked well as well near the apogee. For linking in the perigee region and for LEOs a curved reference motion is suggested.

\section{Acknowledgments}

This work was supported by the Swiss National Science Foundation through grants 200020-109527 and 200020-122070 and the observations from the ESASDT were acquired under ESA/ESOC contracts 15836/01/D/HK and 17835/03/D/HK. 


\section{Appendix A. Examples of Cosmic Filter Results}

The results of the different cosmic filters are shown for some observation series of the night August 25, 2006 of the ESASDT. First all detected object image candidates are displayed in the observation series. In parenthesis first the number of real object images judged by eyesight supported by object image linking algorithms is displayed, secondly the overall number of detected candidates. For the different filters first the number of true object images which are still present after filtering is listed and the total number of candidates after filtering. The true object images are marked with a box. 


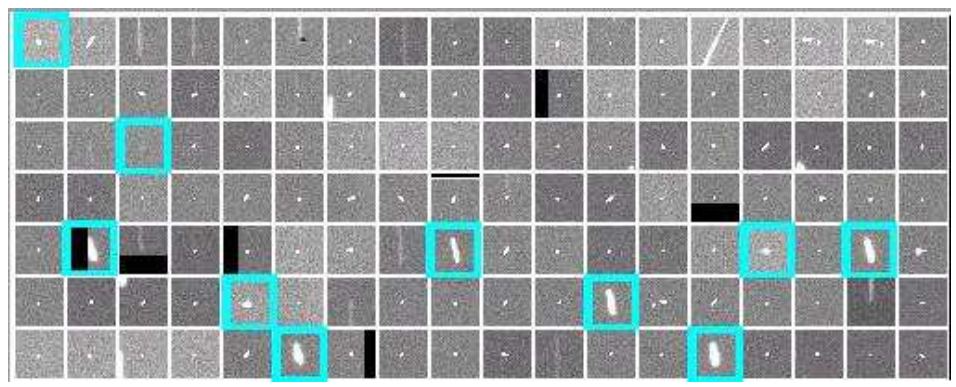

(a) no filter

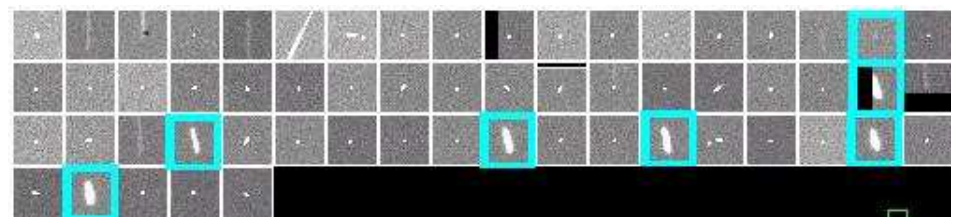

(b) contrast filter

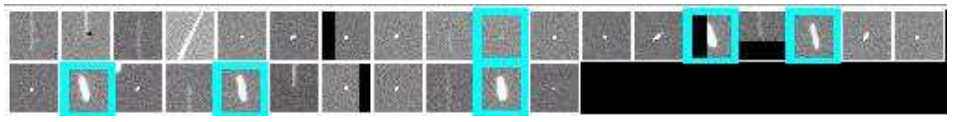

(c) object class filter

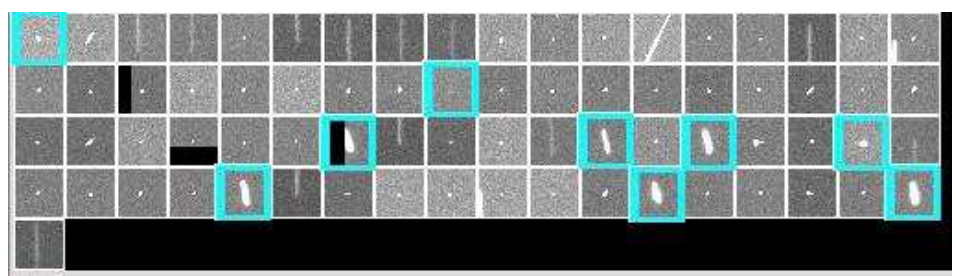

(d) Sobel filter

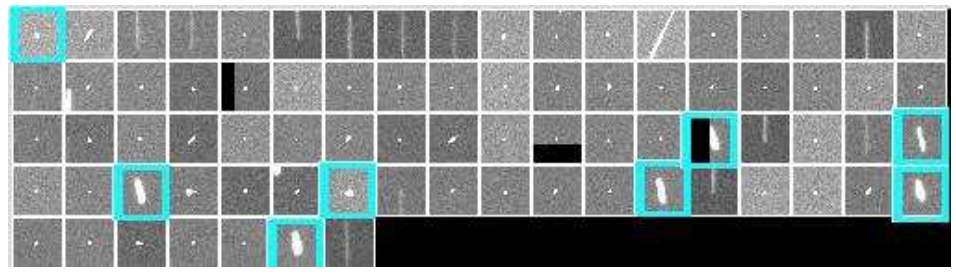

(e) Prewitt filter

Figure A.8: (a) All detected candidates (10/116), (b) contrast filter (7/52), (c) object class filter (6/23), (d) Sobel (9/64), and (e) Prewitt (8/71) edge detection filter: SSO-10A. 


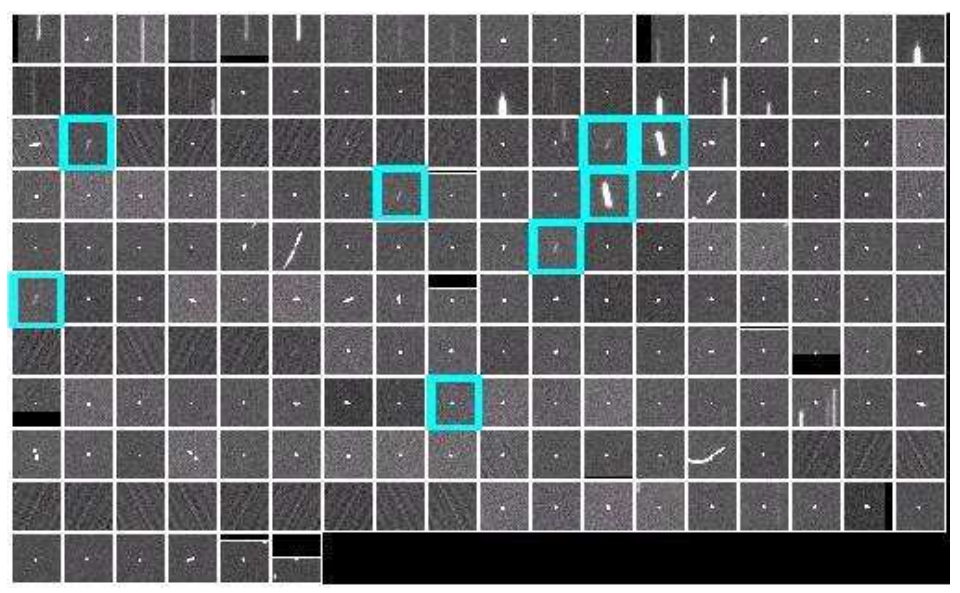

(a) no filter

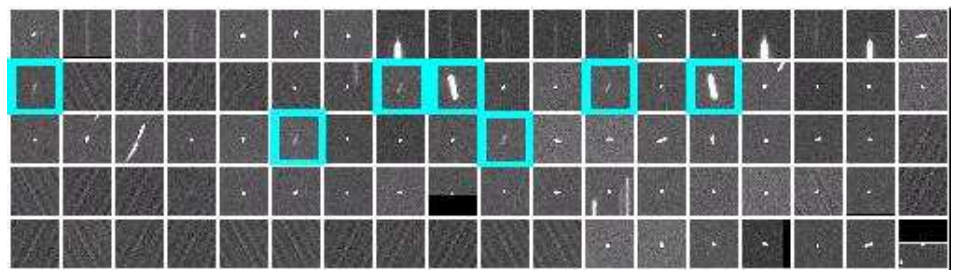

(b) contrast filter

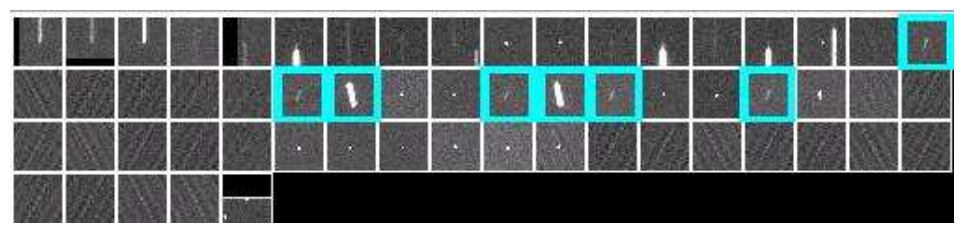

(c) object class filter

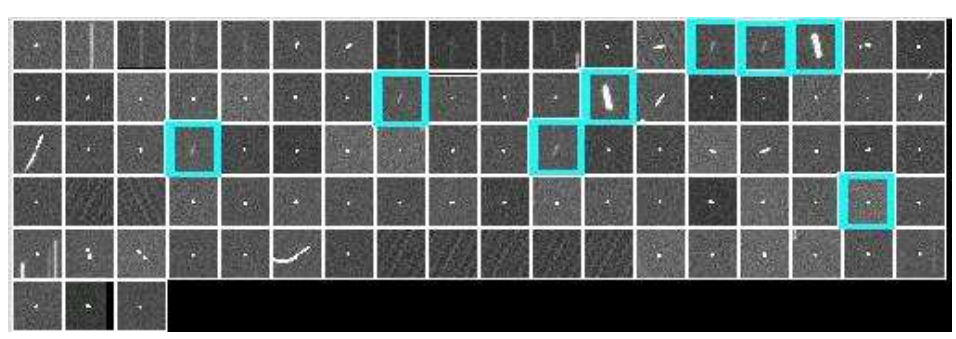

(d) Sobel filter

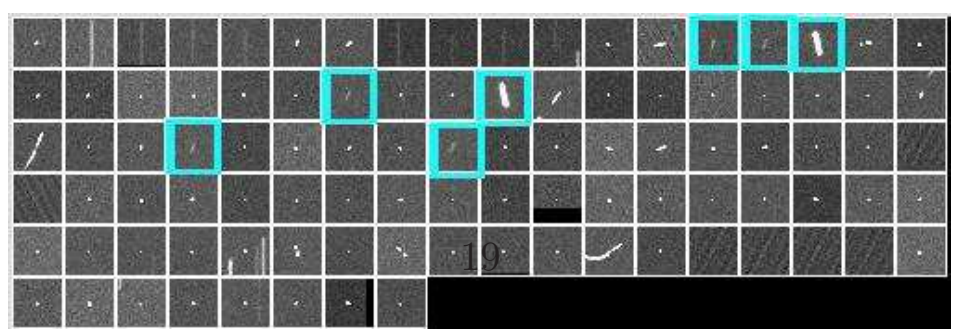

(e) Prewitt filter

Figure A.9: (a) All detected candidates $(8 / 178)$,(b) contrast filter $(7 / 83)$, (c) object class filter (7/52), (d) Sobel (8/85), and (e) Prewitt (7/91) edge detection filter: SSO-27A. 


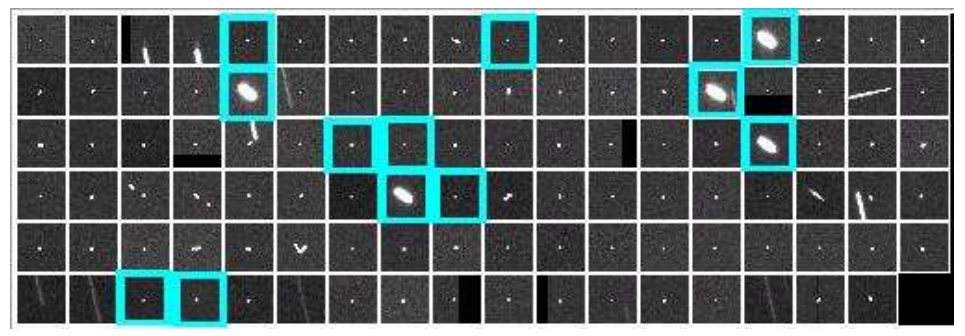

(a) no filter

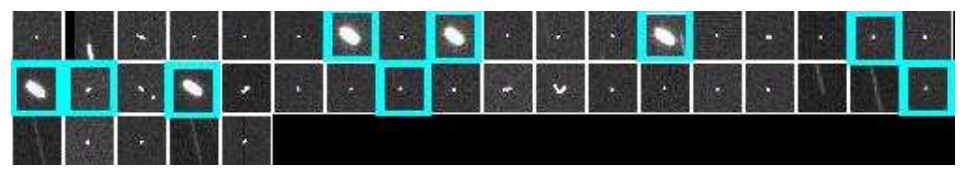

(b) contrast filter

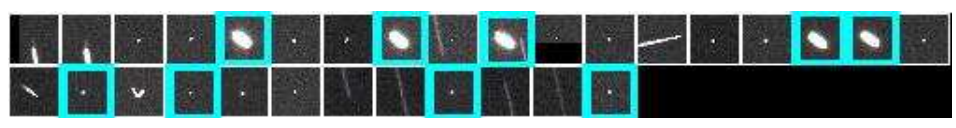

(c) object class filter

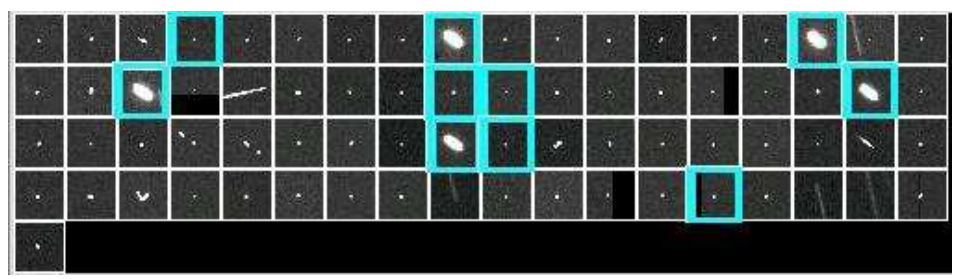

(d) Sobel filter

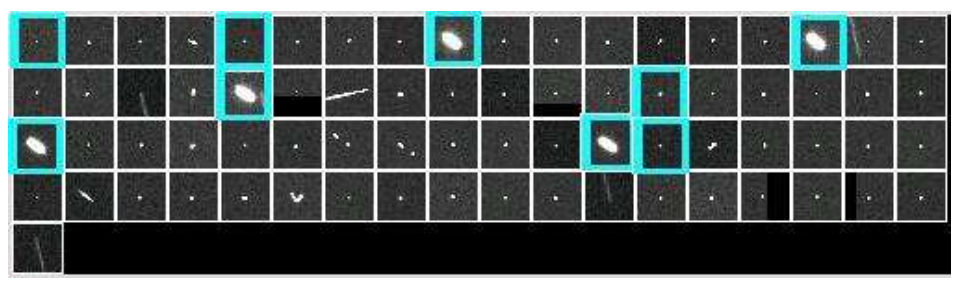

(e) Prewitt filter

Figure A.10: (a) All detected candidates (12/95),(b) contrast filter (9/32), (c) object class filter (9/21)), (d) Sobel (9/63)), and (e) Prewitt (9/64) edge detection filter: SSO-26. 


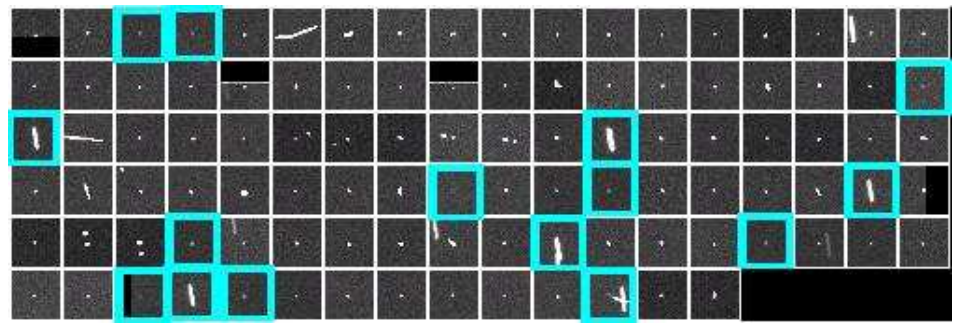

(a) no filter

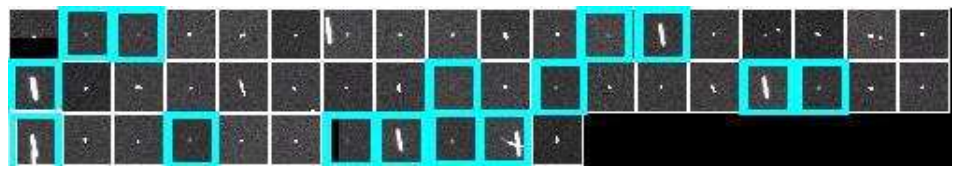

(b) contrast filter

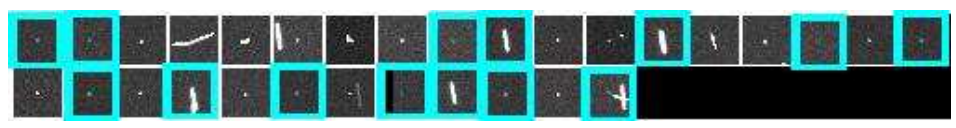

(c) object class filter

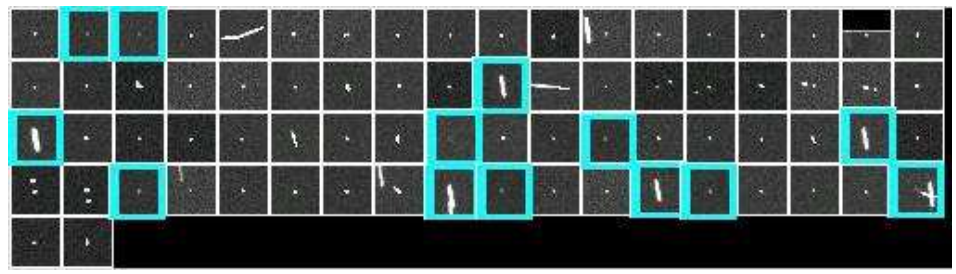

(d) Sobel filter

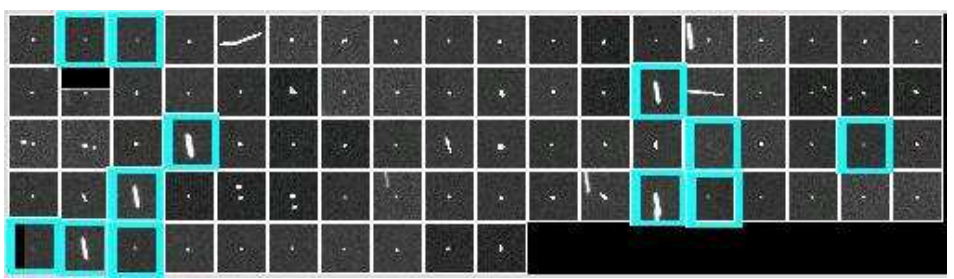

(e) Prewitt filter

Figure A.11: (a) All detected candidates (15/89),(b) contrast filter (15/32), (c) object class filter $(14 / 16)$, (d) Sobel (13/61), and (e) Prewitt (13/69) edge detection filter: SSO-30. 